rateur spécial qui revient plus cher qu'une machine normale. Mais, comme le choix d'une faible fréquence ne s'impose que pour de très grosses unités (cinq périodes pour r.ooo (hevaux) qui justifient l'installation d'un génćratcur spécial, l'augmentation du prix d'achat n'intervient que pour majorer les frais d'amortissement, ce qui ne doit pas entrer en considération, vu le débit énorme de ces fours. Cependant, il serait désirable de pouvoir brancher le primaire d'un four sur un résean à fréquence normale, surtout pour les petils fours deslinés à des usages spéciaux, comme la fonderic de bronze.

Dans ce sens, Röchling-Rodonhauser a réalisé un progrès considérable en ajoutant au four Kjollin un dispositif de chauffage par résistances. D'une part, le canal en forme de huit enveloppanl les deux noyaux, cela permel de réduire la section du bain et d'augmenter sa résistance ohmique. Même avec des chargements de grandeurs croissantes, il n'est pas utile d'augmenter dans la même proportion la section des deux boucles du canal de fusion, l'augmentation de volume se reportant principalcment sur le foyer central. D'autre part, le facteur de puissance est encorc augmenté par le fait qu'une part du courant secondaire circule dans les résistances de chauffage du foyer central. Ces fours peuvent être alimentés à la fréquence 50 , même s'ils atteignent des dimensions très notables, et pour les plus grandes unités, il n'est pas nécessaire de dépasser la fréquence encorc usuelle de vingt-cinq périodes.

\section{(4 suivrè)}

\section{L'ÉLECTRIFICATION DE NOS RÉSEAUX FERRÉS}

Nous avions indiqué à nos lecteurs les efforts faits en vue de remplacer, avec toutes les chances de succès possible, la traction à vapeur par la traction électrique sur nos voies ferrées. Nous avions annoncé le départ d'une mission d'étude, sons la direction de M. Maudurt, Professeur à la Faculté des Sciences de Nancy, pour l'Amérique et nous avions promis de publier le résultat de l'enquite de celte mission : un comple rendu sommaire a été a iñessé au Ministre des Travaux publics et publié dans le Jo:rn. Offic. du s3 août Igrg. Nous le reproduisons ci-dessousin-extenso.

\section{Ministère des Trayaux publics, des Transports et de la Marine marciande}

\section{COMPTE RENDU SOMMAIRE}

adressé au Ministre des Travaux publics par la mission envoyée en Amérique pour l'étude de lélectrification des grands réseaux de chemins de fer.

Le ministre des Travaux publics a instilué, par arrêlé du I4 novembre s 918 , au sein du Conseil supérieur des Travaux. publics, un comité d'études chargé d'examiner les projels présentés par Jos réseaux de Paris-Lyon-Méditerranée, d'OrJéans et du Midi, pom l'alectrification de 10.000 kilomètre chviron de lignes de leurs réseaux.

Ce coinilé, composé de techniciens les plus qualifiés de ladministration et des réseaux de chomins de fer, a cru devoir proposer au ministre d'envoyer aux Etats-Unis une mission d'ingénieurs spécialistes, chargée de recueillir toules les informations relatives aux progrès récents de la traction électrique.

\section{$x^{\circ}$. - Onganisation et composition de la mission}

Cette mission comprenail treize membres, savoir :

Commandant d'Adglards et professeur A. Mauduit, de la Faculté des Scicnces de Nancy, altaché à la direction des chemins de fer, délégués du ministère des travaux publics et des transports :

MM. Pomey, ingénicur en chef des posles el dos télégraphes et Lecorbciller, ingénicur, délćgués de l'administration des postes et des télégraphes ;

MM. Debray, inspecteur, principal, et Barillot, inspecteur, délégués des chemins de fer de l'Etat;

M. Sabouret, ingénicur en chef adjoint à la dircction ;

MM. Balling, ingćnieur principal au service de la voie et Parodi, ingénieur, chef du service électrique, délégués de la compagnie des chemins de fer d'Orléans ;

MM. Japiot, ingénieur en chef du matéricl ol Ferrand, ingénieur au service central du matériel, délégués do la compagnie Paris-Lyon-Médilirranée ;

MM. Bachellery, ingénieur en chef adjoint à la direction, et Leboucher, ingénieur principal à la traction, délégués do la compagnie des chemins de fer du Midi.

La plupart des membres de la mission sont partis le I5 avril pour l'Amérique et sont rentrés à Paris, lc 22 juillet rgrg.

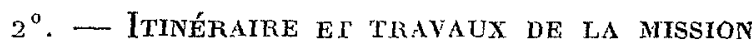

Arrivés à New-York, le 25 avril, nous avous pris contact avec les représentants des diverses usines de construction el compagnics de chemuns de fer et visité les chemins de fur électrifiés suivants :

New-York central, courant conlinu à 600 volts, $3^{e}$ rail ;

New-York New-Haven and Hartford, monophasé à I I.000 volts, 25 périodes;

Pensylvania railroad et Long Island $R$. $R$. continu 600 volts, $3^{\circ}$ rail ;

Chemins de fer de banlieue, assurant cependant un trafic appréciable de marchandises.

Nous avons également visité un certain nombre de centrales électriques puissantes à vapeur, de la Interborough Transit $\mathrm{C}^{\circ}$ et de la Edison Power $\mathrm{C}^{\circ}$ à New-York, ainsi que les centrales du Niagara (hydrauliques) et de Buffalo (à vapeur).

Du 8 au ro mai, visite des atcliers de la General Electric Company (C. E. $\left.\mathrm{C}^{\circ}\right)$ à Schenectady (N. Y.) et discussion avec les principaux ingénjeurs'de cette société, sur les questions concernant l'élcctrification des chemins de fer, en génćral, et notamment l'électrification en courant continu à haulc tension (3.000 volts) du Chicago Milwankee Saint-Paul (7ro kilomèlres en fonctionnement), exécutée par celle société.

Du ix au 25 mai, visite des inslallations suivantes

Electrification du Norfolk and Western railway, en colrant monolriphasé, à i $\mathrm{r} .000$ volts-25 périodes, de Bluefield à Vivian (Virginie).

Electrification du Pensylvania raijroad, en monophrsí $x$ x.000 volts, 25 périodes, de Philadelphie à Paolí.

Chemin de fer électrique Baltimore-Woshinglon-Annapolis, en courant continu r.20o volts (interurbain).

Ateliers de construction de locomolives Baldwin à Philitdelphie.

Ateliers de réparation et de construction du Pensylvania railroad à Altona.

Du 25 au 28 mai, visile des aleliers de la socićté de construction Westinghouse électric and manufacturing company, 
à Pittsburg, el discussion avec les ingénieurs sur l'électrification en général el notamment sur les systèmes monophasés ct monotriphasés installés par celte sociélé, et sur les nouvelles locomolives à courant conlinu 3.000 volts, destinées à l'extension de la portion électrifiće du Chicago Milwaukee Saint-Paul.

Du 29 mai au 4 juin, visites diverses :

Atcliers de construction de locomotives électrıques de la Genial Electric $C^{\circ}$, à Erié.

Sous-stations automatiques de tramways à 600 volts conlinus, modèle Westinghouse ot modèle Général Electric $\mathrm{G}^{\circ}$.

Chemin de fer électrique de Chicago Lake Shore and South Bend, monophasé à 6.000 volts, 25 périodes.

Du 5 au r 4 juin, étude approfondie du Chicago Milwankee Saint-Paul railway, section en exploitation de Harlowton à Avery, 7 10 kilomètres (Montagnes Rocheuses), scclion en cours d'installation de Othello à Tacoma el Scaltle 360 kilomètres (Monlagnes des Cascades), le tout en courant conlinu à 3.000 volts ; ateliers de réparation et dépôt à Deer-Lodge (Montana).

Visite de trois centrales hydrauliques de la Montana Power company, qui foumit le courant, sous forme lriphasée à 100.000 volts et 60 périodes, au chemin de fer élecirique ci-dessus : usine de Rambow, $35.000 \mathrm{kw}$., usine de GreatFalls, $18.000 \mathrm{kw}$. usme de Holter, $48.000 \mathrm{kw}$., toutes les trois sur le Missouri.

La mission s'est disloquée à Sealtle, le 15 juin, el quelques-uns des membres ont continué leur travail en visilan! encore quelques chemins de fer électriques et un certain nombre d'installations de distribution d'énergie à très haut rollage, en vue de recueillir les renscignements nécessaires aux fulures organisations en France.

Ont été ainsi successivement étudiées, les installations ci-dessous :

Central Galifornia traction company, ligne de Slockton à Sacramento ( $7^{2}$ kilom.) en Californie, équipée avec $3^{\ominus}$ rail renversé sous 1.200 volts continus, unique exemple américain de l'application du $3^{\circ}$ rajl à une tension aussi élevée.

léseau du Pacific électric railway, tramways suburbains el interurbains aux environs de Los-Angelos à 600 volts et 1.200 volts continus.

Puget sound light and power company, centrale hydraulique de la While river, près de Seattle (Washington). 48.000 hilowatts, 55.000 volls, chute 130 mètres.

Utah power and lighl company : sous-station principale d'arrivée à Salt Lake Cily (Utah), sous-station extérieure à 120.000 volts, 25.000 kilowalts avec régulation par moteurs synchrones surexcilés (à l'intérieur d'un petit bâtiment spécial.

Great Western power $\mathrm{C}^{\circ}$, de San-Francisco (Californie) : usine hydraulique à Las-Plumas (Californie), sur la Teather river, chute r38 mètres; $65.000 \mathrm{kw}$. II5.000 volts ; ligne doublc, sur pylônes uniques, de 246 kilomètres ; sous-station d'arrivée à Oakland.

Pacific gas and electric company, à San-Francisco, usine à yapeur avec chaudières chauffées à l'huile, $57.000 \mathrm{kw}$.

Southern Galifornia Edison company de Los-Angeles : deux usines hydrauliques voisines, à Big-Creek, dans la Sierra-Nevda, de chacine $28.000 \mathrm{kw} ., 600$ mètres de chute, voltage : 150.000 à $\mathrm{I} 60.000$ volts; deux lignes en aluminium et acier, sur pylónes séparés de 400 kilomètres de longueur ; sous-station d'arrivée à Eagle-Rock, près de Los-Angeles, à rho.0no volts. avec régulation par moteurs synchrones.

\section{$3^{\circ}$. - Renseignements principaux Recueillis AU COURS DE LA MISSION}

Indépendammenl du rôle général de la mission, consistan à recueillir tous documents utiles sur l'électrıfication des chemins de fer et sur la distribution de l'énergie électrique à haute tension, le but principal de cette mission était de rechercher, en tolahsant les renseignements fournis par l'étude des chemins de fer électrıques suisses et italiens, d'une part, et américains, d'autre part, s'il existait un système de traction élcclrique pour grandes lignes nettement supérıeur aux autres et susceptible d'être adopté, à l'exclusion des autres, par toutes les différentes compagnies intéressées, poúr les électrifications projetées dans le centre et le sud de la France.

Des quatre systèmes de traction électrique de grandes lignes, actuellement en fonctionnement dans le monde, savoir : monophasé, triphasé, monotriphasé et continu à haute lension, Ic triphasé avait pu déjà être étudié en détail en Italie où il est employé en grand, tandis qu'il n'est utilisé d'une façon appréciable en aucune autre contrée, et le monophasé avait été examiné également en fonctionnement en France sur les chemins de fer du Midi el en Suisse à la compagnie du Lotochberg, et, en projet, près des chemins de fer fédéraux suisses qui ont adopté ce système pour l'électrification progressive de-tout leur réseau, électrification actuellement à l'étude et même en cours d'exécution pour lc chemin de fer du Gothard.

Seuls, le monotriphasé el le continu à haute lension n'cxistent qu'en Amérique et devaient faire l'objel principal. des travaux de la mission. En même temps, l'examen des installations américaines en monophasé (à 25 périodes, alors que les installations analogues françaises sont à 16 périodes) permettraient de compléter l'étude du monophasć.

Les renseignements de loute nature recueillis en Amérique feront l'objet d'un rapport délaullé de M. Mauduit, rapport qui sera terminé au courant de ce mois et scra soumis à la sous-commission lechnique dès le début d'octobre, pour servir de base à la discussion d'une proposition tendant à fairo choix d'un système de traction unique pour les différentes compagnies, suivant une formule à élablir frar celte sousrommission avec approbation du comilé tout cnticr.

Le but de ce compte rendu sommaire est de donner seulcment les résultats les plus importants el les impressions principales qui se dégagent de l'expérience américaine, avec les conclusions personnelles du rapporteur : les documents ont été recueillis par tous les membres de la mission, tantôt simultanément, tantôt séparément, mais les opinions érnises dans cette note, bien qu'elles soient en général l'écho des impressions générales de la mission, sont des opinions personnelles et n'engagent que le rapporteur, tant qu'elles n'auront pas été approuvées par la sous-commission technique, en présence de tous les membres de la mission, appelés devant cette commission pour les compléter et les discuter.

\section{$4^{\circ}$. - Etectrification eN MONOPHaSÉ}

Les principales lignes équipées en monophasé (à 25 pério. des et I 1.000 volts) sont le New-York, New-Haven and Hartford railroad et le Pensylvania railroad (Philadelphie à Paoli). Bien que ces lignes soient des lignes de banlieuc, elles sont intéressantes à étudier, parer que le système de traction employé est applicable aux grandes lignes el analogue à celui du Midi français, sauf la fréquence ; 25 périodes au lieu de $\mathbf{r} 6$. 
New-York, New-Haven and Hartford railroad (102 kilomètres de route élcctrifiée).

L'électrification de ce réseau a été déterminée par l'ordre de l'Etat de New-York; clle comprend une partie en courant continu à 600 volts avec $3^{\mathrm{c}}$ rail renversé, sur un tronçon commun avec le New-York central railroad, au départ de NewYork.

Sa partie extérieure est en monophasé à y r.0oo volts, avec fil aérien de contact. La nécessité de fonctionner, soit en continu 600 volts, soil en monophasé a r.ooo volts, complique beaucoup l'équipement des loconotives qui doivent pénétrer dans la ville de New-York.

Le trafic est important et le fonctionnement technıque convenable, après qu'on a eu à surmonter beaucoup de difficultés dans les premières années. Ces difficultés ont surtout consisté dans la lutle contre les accidents dus aux courtscircuits survenant fréquemment sur la ligne de contaćt ou sur les feeders d'alimentation, et contre les perturbations provoquées dans les lignes télégràphiques cl téléphoniques voisines, appartenanl, soit au réseau, soit à des compagnies différentes.

On est arrivé à résoudre les problèmes ainsi posés, mais au prix d'organisations complexes, délicates et onéreuses d'installation et d'entrelien. Les lignes téléphoniques ont été mises en câbles sous plomb et enterrées ; la distribution du courant a été faite sous 22.000 volts, au moyen de 30 auto-transformateurs compensateurs répartis sur les xo2 kilomètres de route, pour diminuer la chute de lension trop élevée dans les lignes, et réduire les influences sur les lignes télégraphiques et téléphoniques : ce dispositif remplace les transformateurs-suceurs du Midi français, avec l'avantage supplémentaire de la réduction de la chute de tension.

Le parc comprend ro 3 locomotives et 26 automotrices ; les frais d'entretien sont relativement élevés el le personnel des ateliers de réparation assez nombreux.

Les moteurs monophasés sont délicats et demandent une surveillance dủ collecteur assez soignée.

Pensylvania Railroad (lignes de Philadelphie à Paoli, $32 \mathrm{~km}$. à 4 voies, et de North Philadelphia à Chesnut Hill, $20 \mathrm{~km}$. à 2 voies).

Le parc ne comprend que des automotrices, pas de locomotives et le service est du type banlieue à gros trafic.

Le fonctionnement technique est bon, les motcurs n'étant pas assujettis à fonctionner à la fois sur courant continu et courant thonophasé, sont d'un type plus moderne, avec meilleure commutation.

Des précautions spéciales sunt prises contre les courtscircuits, el la lutte contre les influences sur les lignes télégraphiques et téléphoniques a été résolue d'une façon suffisante par la mise de ces lignes en câbles sous plomb enterrés; l'emploi de transformaleurs d'alimentation rapprochés ( 5 pour $52 \mathrm{~km}$. de route) et de transformaleurs-suceurs intercalés dans la voie à des distances très courtes en certains endroits (de l'ordre du kilomètre).

En marche normale, le fonctionnement des lignes de signalisation est suffisant, mais les courts-circuits, assez rares d'ailleurs, produisent des perturbations importantes : un dispositif enregistreur très inléressant,branché sur un fil témoin placé dans un câble, permet de contrôler à chaque moment le voltage perturbateur induit dans les lignes télégraphiques et téléphoniques.

Les installations de fraction américaine en courant monophasé, spécialement à cause de la fréquence ćlevée adoptée (25 périodes par seconde au lieu de 16 périodes en Europe), l'réquence qui a été imposée par les conditions locales pour utiliser directement les nombreux réseaux de distribution it celte fréquence, el de l'emploi de moteurs souvent un pru moins bons que ceux que nous avons rencontrés au Midi of en Suisse, représentent un stade plutôt moins perfectıonnć que les installations similaires d'Europe.

Toutefois, la lutte contre les perturbations dans les lignes téléphoniques et télégraphiques y a élé poussé à un degré do perfection considérable, et il y aurail certainement lieu d'en tenir le plus grand compte, si l'on adoptait, en France, ce système de traction. D'autre parl, les lignes de contact à suspension calénaire sont d'une exéculion remarquable.

Si nous totalisons manntenant l'expérience de France, de Suisse et d'Amérique, nous sommes amenés à conclure que le système monophasé est loin d'ètre au point et préscnle encore un certain nombre de problèmes, insuffisamment résolus dans la pratıque actuelle, notamment la réalisation d'un moteur susceptible de rester suffisamment longtemps sous courant sans tourner, pour pouvoir démarrer des trains lourds dans des rampes importantes et celle du f́reinage électrique avec récupération.

De plus, il conduit à des complications importantes pour la protection des circuits téléphoniques voisins, ce qui augmente notablement les frais d'installation, qui, sans cette considération, seraient inférieurs nettement à ceux qu'occasionnent les systèmes triphasés et continu à haute tension.

Les dépenses d'entretion du matéricl tractcur se sont toujours montrées plus élevées que dans ces deux systèmes el les moteurs sont moins robustes et susceptibles de moindres surcharges.

\section{$5^{\circ}$. - Electrification eN Monotriphasé}

Dans le système monotriphasé, que les Américains appelleni "splitphase ", le courant est foumi au fil de contact, unique comme dans le monophasé avec retour par les rails, sous forme monophasée, mais il est transformé sur la locomotive, au moyen d'un convertisseur spécial, en courants triphasés, et les moteurs, utilisés sur cetle dernière, sont des moteurs d'induction triphasés.

Le but de cetto disposition est de profiter à la fois du fil de contact unique du système monophasé (alors que le triphasé italien nécessite deux fils de contact aériens, en plus du rail servant de retour) et du moteur d'induction triphasé, robusle et économique, susceptible de rester sous courant sans tourner pendant plusieur's minutes et d'assurer ainsi le dérnarrage des trains les plus lourds, ce qu'on n'a pu, jusqu'ici, obtenir avec le moteur monophasé ordinaire à collecleur.

Il 'n'existe, à l'heure actuelle, qu'une ligne fonctionnant avec ce système : c'est la ligne de Blueflicld à Vivian, du Norfolk and Western Railway, dans les montagnes Apalachiennes, en Virginie et Ouest-Virginie, sur une longueur de 48 kilomètres, avec voie double ou triple, nombreuses courbes el rampes atteignant.20 millimètres par mètre.

Une locomotive électrique y remorque des trains de 3.000 tonnes, principalement chargés de charbon, à la vitesse de 22 kilomètres 50 à l'heure, avec machine de renfort en queue, pour les rampes dépassant 15 mullimètres par mètre.

Ces locomotives sont souples et robustes, mais leur fonctionnement présente divers défauts mécaniques et électriques qui n'ont pu être corrigés jusqu'ici d'une façon suffisante et font que cette installation ne peut être considérée encore que comme en période d'essai et que les dépenses d'entretien du matériel tracteur sont plus élcvécs qute dans les antres systèmes.

Au point de vue mécanique, la transmission du mouvement des moteurs aux essicux, qui se fait par faux essieu et 
biclles horizontales, occasionne une usure rapide des coussinets et même une dislocalion du châssis ou des ruptures de bielles, par suite des eflorts énormes déyeloppés lors des déplacements verlicaux du châssis.

Au poinl de vue électrique, les principaux inconvénients sont les suivants :

Le triphasé produit par le convertisscur actuel n'est pas parfaitement symétrique et les phases ne sont pas parcourucs par des courants égaux ; d'autre part, les rotors des moteurs étant fermés sur des rhéostats liquides différents, les charges ne sont jamais également réparties entre les moteurs, avec des écarts souvent considérables. Il est bien prévu un réglage de ces charges à la portée du mécanicien, mais ce dernier, très occupé, n'assure pratiquement qu'un équilibrage très imparfail et les moteurs, souvent surmenés, se détériorent ussez rapidement.

Lc facteur de puissance esl très bas, par suite de la présence du convertisseur d'induction qui ajoule ses dépenses d'amanlation à colle des motcurs.

Pour remédier à ces divers défauts, sauf celui de la répar lition de charge entre les moleurs, le constructeur étudie en ce moment l'utilisation d'un convertisseur synchrone, à bon lacteur de puissance et à triphasé plus symétrique, mais il n a pas encore été fait dapplication pratique de ce nouvel appareil pour lequel on peut craindre une grande instabilité en présence des à-coups dans la ligne de contact.

P'ar suite des nombreuses réparations cn cours et du manque de locomotives électriques du fail de la guerre, l'exploitation du troncon Blueffield-Vivian ullise encore de nombreuses locomotives à vapeur.

Le Pennsylvania Railroad étudie, de son còté, une applicalion du monotriphasé à la ligne à quatre voies d'Altoona à Johnstown, sur la ligne de Philadelphie à Chicago. Une locomolive d'essai est en cours d'expérimentation, mais aucune Installation fixe n'est commencée sur la voie.

En résumé, le monotriphasé, donl le principe semblait, à première vue très intéressant et qui pouvait apporter une aide cllicace au monophasé par l'emploi de locomotives ou automotrices en monophasé direct pour les trains rapides ou légers, et de locomotives monotriphasées pour les trains lourds et lents, toutes ces locomótives élant alimentées par le même fil de contact en couranl monophasé, s'est trouvé présenter en pratique de nombreux défauts qui n'ont pu être cncorc corrigés, et fonl que ce système n'a pas répondu aux cspérances qu'il avait fail naître.

\section{$6^{\circ}$. - EtectRIFICATION EN CONTINU A IMAUTE TENSION}

Depuis longtemps déà, le courant continu à 600 volts est uhlisé d'une façon- " standard ", pour la traclion électrique urbaine el suburbainc, soil avec fil aéricn pour les tramways. soil avec $3^{\mathrm{e}}$ rail pour les chemins de fer de banlieue (ligne des Invalides à Versailles et de Paris à Juvisy, Mélropolıtain).

Aux Etats-Unis, la plupart des lignes interurbaines fonclionmenl en courant continu à r.200 volts, avec fil aérien ; un nombre important de ces lignes sont de véritables chemins de fer, avec lrafic de marchandises et de voyageurs, alleignanl des vitesses de 60 à 80 kilomètres à l'heure ; beàucoup d'entre clles, équipées originairement en monophasé à des tensions comprises entre 3.000 volts et 6.000 volts, ont été transformées en continu r.200 volts. Le matériel correspondant à ce voltage est maintenanl aussi standardisé que celui des tramways à 600 volts.

Encouragés par l'excellent fonctionnement de ces installations à I.200 volts, les Américains ont essayé, avcc plein succès, d'élever la tension continue à 2.400 volts et ont équipé dinsi la ligne minière de Butte à Anaconda du Butte Anaconda and Pacific Railway (Etat de Montana), 53 kilomètres à roie unique; ensuite a élé exécutée à 3.000 volts, la plus grande électrification du monde, de Harlowton à Avery, 7 lo kilomètres, à simple voie, à travers les montagnes Rocheuses el la région de Missoula, sur le Chicago Milwankee and Saint-Paul Railway.

L'électrification dun second tronçon de 360 kilometres, entre Othello el Tacoma-Seattle, jusqu'au Pacifique, est en cours d'exécution et fonctionnera avant la fin de l'année, et celle de la portion comprise entre Avery el Othello, environ de la même longueur, est dès maintenant décidée.

Nous avons étudié avec un soin particulier cette inslallalion du Chicago Milwankee and Saint-Paul ; et tous les membres de la mission ont été unanimes à reconnaître que cette ćlectrification, de beaucoup la plus importante du monde, élail en même temps très supérieure à toutes les autres, par l'excellence de son fonctionnement technique, à tous les points de vue.

L'énergie électrique est fournie par la Montana Power Company, sous forme de courants triphasés à Io0.000 volts, et transformćc en courant continu à 3.000 volts, dans des sous-stations rotatives, comprenant des moteurs générateurs, qui sont constitués par un moteur synchrone et deux dynamos à courant continu, montées sur le même arbre et accouplées électriquement en série, de façon à produire chacune r.50o volts seulement sur son collecteur.

Ces sous-stations sont la partie la plus délicate el la plus onéreuse de ce système de traction ; mais elles sont au nombre de quatorze seulemenl pour 7 ro kilomètres (environ tous les 50 kilomètres) et ont un fonctionnement cxcellent. Elles nécessitent seulement chacune un personnel de trois hommes) un chef et deux aides pour une marche permanente, avec une puissance de 4.000 ou 6.000 kilowalts. Par l'emploi de disposilifs prolecteurs sur les collecteurs, et d'interrupteurs cxtra-rapides dans le circuit général, on est arrivé ì supprimer les accidents résullanl du phénomène le plus redoutable avec le courant continu : le coup de feu au collecteur (appelé souvent flash ou flache), en cas de courtcircuit.

L'excellente mise au point de l'organisalion de ces sousstations est pour beaucoup dans le succès obtenu par le comrant continu à haute lension.

A la tension relalivement faible de 3.000 volts sur la ligne de contact (au licu de 1.000 à 15.000 volts, pour le monophasé) correspond une grande intensité de courant à capter pour la traction des trains Iomds. L'expérience a montré qu'avec un fil de conlact double, et un archet panthographe a double sabot el quadruple contact, on capte facilement .500 à 2.000 ampères à la vilesse de 80 à 96 kilomètres à l'heure, et 4.000 ampère à ]a vitesse de 25 kilomòtres à l'heurc, ce qui est plus que suffisant pour les trains les plus lourds et les puissances les plus grandes.

Les locomotives sont d'une grande facilité de conduile et d'un fonctionnement parfail, le moteur série à courant conlinu étant d'ailleurs le moteur idéal pour la tráction, comme l'a depuis longtemps montré l'expérience, de tramways et chemins de fer électriques de banlieue. Elles sont pourvues d'un froinage élcctriquc avec récupération, merveilleusement réglable, qui assure la marche la plus souple dans les descentes ct occasionne une ćconomie importante de courant et surtoul de bandages el de sabots de frein. Un seul bobineur, avec un aide, assure l'entretien des 336 moteurs des 42 locomtives en service; l'ancien dépôt de locomotives à vapeur de Decr Lodge, correspondant a 360 kilometres de 
lignes, a suffi largement pour l'installation du dépôt des locomotives électriques et des ateliers de réparation, pour la longueur tolale électrifiée, soit 7 ro kilomètres.

Une seule locomotive remorque les trains de voyageurs de 900 à r.ooo tonnes américaincs (1) même dans les rampes de 20 millimètres par mètre; les trains de marchandises de .8oo tonnes américaines sont traînés par une seule locomotive dans les rampes de 10 millimètres (l'effort de traction est alors de 32,8 tonnes métriques) et par deux locomotives pour les pentes supérieures. Le poids remorqué moyen par train de marchandises est d'environ I.90o tonnes américaines. Dans les trains comportant deux locomotives, la seconde machine est placée au milieu du train et non à la queue ; il faut d'ailleur's remarquer que la dérive n'est pas à craindre en Amérique, tous les trains de marchandises, comme ceux de voyageurs, étant munis du frein automatique à air comprimé, sur tous les wagons.

Un avanlage considérable du système de traction à courant continu est qui'l ne semble apporter que des perturbations insignifiantes aux lignes télégraphiques et téléphoniques; nous avons pu nous rendre compte qu'on téléphonait fort bien sur les lignes de service du chemin de fer placées tout le long de la voie en fil aérien, sans appareil de protection.

Un appareil télégraphique imprimeur multiplex, faisant le service entre Spokane ct Helena à travers un circuit à retour par la terre, détourné spécialement pour nous, de façon à emprunter un fil placé sur les poteaux du chemin de fer électrique sur une longueur de 270 kilomètres, a fonctionné parfaitement pendant huit jours, sans même être troublé par trois courts-circuits francs faits intentionnellement, entre le fil de contact et le rail, sur le trajet du fil télégraphique.

Malgré la perte d'énergie due à la transformation du courant triphasé en courant continu dans des sous-stations rotatives tournant d'une façon permanente, quelle que soit la charge, alors que le nombre journalier de trains est assez réduit, savoir : deux trains de voyageurs et trois à quatre trains de marchandises dans chaque sens, le rendement de la traction est bon, 27 watt-heures par tonne métrique-kilomètre remorquée, ce qui correspond à un rendement global de 50 p. roo depuis l'énergie achelée au producteur jusqu'au crochet d'attelage.

\section{$7^{\circ}$. - Conglusions Relatives au Choix d'un système} DE TRACTION ÉLECTRIQUE

Devant les résultats remarquables oblenus par le Chicago Milwankec Saint-Paul, en courant continu à 3 .ooo volts, le rapporteur n'hésite pas à conclure formellement en faveur de l'adoption de ce système, qu'il estime être actuellement le seul véritablement au point, pour la traction électrique des grandes lignes.

Il est possible qu'avec le monophasé qui présente, à première vue, l'avantage de se prêter à une grande variété de combinaisons, on arrive un jour à un fonctionnement satisfaisant, mais il est hors de doute que la pratique actuelle est loin de cet état désirable de mise au point.

Le courant continu présente l'inconvénient d'être un peu plus onéreux de première installation, à cause des sousstations rotatives destinées à transformer le courant triphasé à 50 périodes généralement produjt dans les centrales ; toutefois, il faut remarquer que, pour bénéficier d'une économie à cet ćgard avec le monophasé, il faut engendrer directement ce courant monophasé à faible fréquence (x 6 périodes) au moyen de groupes électrogènes spéciaux, faute de quoi, si l'on veut utiliser le courant produit normalement par les centrales (triphasé à 50 périodes), il faut recourir à la transformation rolative aussi bien avec le monophasé qu'avec le continu. A ce poinl de vue, le courant conlinu ofle l'avantage de se prèter à l'utilısation du courant de n'importe quelle centrale, dans les mêmes conditions.

En ce qui concerne la dépense d'exploitation, des calculs complets et précis faits par les services techniques des compagnies pourront seuls élablir la comparaison entre les divers systèmes; he rapportcur estime loutcfois que l'écart ne sera pas considérable el n'aura pas à entrer en ligne de comple pour le choix du système.

L'absence presque complète de perturbations sur les lignes léléphoniques et lélígraphiques constitue, pour le comranl continu, une supériorité très considérable sur les autres systèmes.

Nous n'avons pas parlé du triphasé qui n’a en Amérique qu'une application locale insignifiante ; malgré certains avantages obtenus par les Italiens, nous sommes d'avis do le rejeter, spécialement par suite de la complication el dı prix élevé d'installation et d'cntretien de ses deux grandes lignes de contact.

\section{$8^{\circ}$. - Consibérations Économiques sur la tracionon ÉLECTRIQUE}

Au point de vue économique, les documents que nous rapportons d'Amérique sont beaucoup moins complets el moins précis que les renscignements techniques.

D'autre part, il est nécessairc, pour conclure de l'expérience américaine à l'avenir économique de la traction électrique européenne, de faire subir aux chiffres des modifications considérables, à cause des deux principales conslanics quí différencient l'exploitation américaine de l'exploilation européenne :

$I^{\circ}$ En Amérique, l'attelage employé a une résistance à la rupture d'environ $\mathrm{r} 35$ tonnes, et on peut admettre des efforls de traction allant jusqu'à fo tonnes ; en Europe, les attelages sont de deux modèles, dont les résitançes à la rupture sont respectivement 35 et 55 tonnes, et les "efforts de traction admis sont limités à ro tonnes (exceptionnellement 12 ì I5 tonnes, en Suisse) ;

$2^{\circ}$ En Amérique, tous les wagons de voyageurs el de marchandises sont munis du frein à air comprimé.

Il résulte de là qu'on emploie en Amérique des loconotives deux à trois fois plus puissantes, des trains de marchandises deux à trois fois plus longs et plus. Iourds qu'cn Europe, et que le personnel de conduile des trains de marchandises est relativement beaucoup moins nombreux, ce qui modifie complètement les dépenses d'exploilalion.

Des calculs précis faits par les compagnies et surtoul les résullats des premières électrificalions exécutées et la considération des prix exacts du charbon, pourront seuls permcltre de savoir dans quelles conditions la traction électrique sera plus ćconomique que la traction à vapeur ; on sait déjà, d'ailleurs̃, que l'économie sera surtout sensible sur les lignes à gràndes déclivités et à grand trafic el il est probable que pour beaucoup de lignes s'écartant par trop de ces conditions, la traction électrique sera plus onéreuse que la traction à vapeur.

Toutefois, la nécessité, de plus en plus importante, d'économiser le charbon et les grands avanlages accossoires bien connus de l'ćlectrification rendent nécessairc l'exécution la plus rapide possible des premiers travaux en vue de l'électrificalion progressive des lignes les plus inléressantes de nos réscaux du P. O., du P.-I..-M. et du Midi.

A. Maudutr,

Professeur d'Eleclrolechnique à l'Université de Nancy. 